\title{
Experimental study of imperfect phase synchronization in the forced Lorenz system
}

\author{
Antonio Pujol-Peréa) \\ Instituto Mediterráneo de Estudios Avanzados, IMEDEA (CSIC-UIB), E-07071 Palma de Mallorca, Spain \\ Oscar Calvo ${ }^{\text {b) }}$ \\ Departament de Física, Universitat de les Illes Balears, E-07071 Palma de Mallorca, Spain \\ Manuel A. Matías ${ }^{\mathrm{c}}$ \\ Instituto Mediterráneo de Estudios Avanzados, IMEDEA (CSIC-UIB), E-07071 Palma de Mallorca, Spain \\ Jürgen Kurths ${ }^{\text {d) }}$ \\ Institute of Physics, Potsdam University, PF 601553, D-14115 Potsdam, Germany
}

(Received 14 May 2002; accepted 30 September 2002; published 21 February 2003)

\begin{abstract}
In this work we demonstrate for an experimental system, that exhibits the Lorenz butterfly attractor behavior, that perfect chaotic phase synchronization cannot be achieved in systems with an unbounded distribution of intrinsic time scales. Instead, imperfect phase synchronization is characterized by the occurrence of phase slips, associated to epochs of time during which the chaotic oscillator exhibits a slower time scale. Interestingly, during phase slips the chaotic oscillator keeps in sync with the drive, but with a different locking ratio. (c) 2003 American Institute of Physics. [DOI: $10.1063 / 1.1525126]$
\end{abstract}

A class of (so-called phase coherent) chaotic oscillators, namely Rössler oscillator, has been shown to exhibit phase synchronization in the case that the oscillator is driven by a sinusoidal generator (and also in the case of two, slightly detuned, chaotic oscillators). This behavior is characterized by an approximately constant relationship between a suitably defined phase for the chaotic oscillator and the phase of the sinusoidal generator. Interestingly, the oscillator remains chaotic, and so does the amplitude, while its rhythm is dictated by the external sinusoidal generator, and, thus, is much more regular. Quite different is the case of chaotic oscillators for which a saddle equilibrium belongs to the attractor, as is the case of the Lorenz oscillator. The most relevant feature of this type of systems is that a typical trajectory in phase space has some probability of passing close enough to the stable manifold of the saddle point (in the Lorenz system this happens whenever a trajectory changes lobe). The closer the trajectory approaches the stable manifold of the saddle point, the longer is the return time, i.e., the time needed to perform a turn. Ultimately, these extra long return times (compared to the typical return times of the system, and also to the period of the external sinusoidal generator) make it difficult to achieve the state of (perfect) phase synchronization, leading to the behavior known as imperfect phase synchronization. Here we shall demonstrate how this behavior is typical, in the sense that it can be easily reproduced in an experimental implementation of the Lorenz oscillator.

a)URL; http://www.imedea.uib.es/PhysDept/

b)Electronic mail: oscar@galiota.uib.es

${ }^{c}$ Electronic mail: manuel@imedea.uib.es

${ }^{\mathrm{d})}$ Electronic mail: juergen@agnld.uni-potsdam.de

\section{INTRODUCTION}

Recently, there has been a lot of interest in the study of manifestations of synchronization in several physical, chemical, biological, and technological systems. ${ }^{1}$ Probably the simplest (and most studied) situation corresponds to a (dynamical) system forced by a sinusoidal generator. In this context, synchronization is understood as the readjustment in the rhythm of the forced system under the influence of the driving signal. In the periodic case this was already studied by Arnold, and then by many others (see, e.g., Ref. 2), and the main features of this behavior have been uncovered. In particular, as the coupling becomes different from zero one expects regions of parameters for which synchronization (or phase locking) occurs. If one represents the amplitude versus the frequency, both corresponding to the sinusoidal forcing, one obtains the well-known Arnold tongues, namely wedgelike regions of synchronized behavior.

The situation is somehow more complex if one considers systems with chaotic behavior. For relatively strong coupling, it was already shown ${ }^{3,4}$ that one may have complete synchronization between identical, uni- or bi-directionally coupled chaotic oscillators. Generalized synchronization, ${ }^{5}$ implying a functional relationship between drive and response, has been also found for uni-directionally coupled chaotic systems. More recently a type of partial synchronization was shown for bi-directionally (slightly detuned) coupled oscillators: phase synchronization. ${ }^{6}$ The chaotic systems that have been shown to exhibit this behavior (e.g., Rössler system ${ }^{7}$ ) can be considered as true oscillators, in the sense that the systems exhibit oscillations in phase space (around some center of oscillation). This implies also that a phase variable can be suitably defined, ${ }^{8,9}$ and the observed behavior is that there is some regime in which the two systems share the phase (apart from a constant, smaller than 
$2 \pi$ ), while the amplitudes vary chaotically and are practically uncorrelated. ${ }^{6}$ The concept of phase synchronization has shown to be useful, although it cannot be applied in general for an arbitrary dynamical system, and, in particular, allows to study synchronization behaviors where not much information can be obtained by looking at correlations between the coupled systems.

Phase synchronization has also been found in the case of sinusoidally forced chaotic oscillators, and this will be the focus of our study. The chaotic oscillator and the drive are not homologous, but it can be shown that one may get phase synchronization, ${ }^{9}$ in the sense that a suitably defined phase for the chaotic oscillator minus the phase of the drive are bounded by $2 \pi$. Chaotic systems with low phase diffusion ${ }^{10}$ (e.g., the Rössler system) exhibit, in principle, perfect phase synchronization behavior under sinusoidal forcing. Quite different is the situation if one works with the Lorenz ${ }^{11}$ system (at the parameter values for which it exhibits the well known butterfly attractor). For the butterfly Lorenz system the saddle equilibrium point at the origin is part of the closure of the attractor, and makes the attractor nonhyperbolic by inducing singularities for the return maps. In particular, the return times to a suitably defined Poincaré cross section will exhibit a singularity, corresponding to the crossing of the return map with the stable manifold of the saddle equilibrium, that happens sometimes when the Lorenz system changes lobe.

Thus, it is normal to expect that phase synchronization will not be perfect for a driven Lorenz system, in the sense, that the system will not be able to follow the pace of the drive at all time, namely when passing close to the saddle equilibrium. This has been, indeed, recently shown through numerical simulation and theoretical arguments by Zaks et al. ${ }^{12-14}$ This imperfect phase synchronization manifests, among other effects, in the presence of phase slips, that are jumps by $2 \pi$ in the phase. It must be pointed out that phase slips may also be obtained in, at least, two other different circumstances, namely in the presence of noise, and for parameter values close to the onset of phase synchronization. In the first case the stochastic (high-dimensional) degrees of freedom may induce occasional kicks out of the synchronized state leading to some kind of higher-dimensional behavior, while in the second the phase locked stable and unstable solutions, respectively, will collide leading to a socalled eyelet intermittency. ${ }^{15}$ Instead, imperfect phase synchronization is a behavior in which a purely deterministic system also exhibits a nonuniform phase dynamics, not associated to external influences or the proximity to the onset of phase synchronization.

An alternative way of understanding phase synchronization is in terms of unstable periodic orbits (UPOs). ${ }^{16}$ In the case of phase coherent systems (systems with a relatively narrow distribution of return times, i.e., of frequencies), phase synchronization is attained when all the UPOs become entrained with the forcing (around the natural frequency of the system), and this is possible for all the UPOs simultaneously as they have similar frequencies. In the case of the Lorenz butterfly system (and in general systems with a broad distribution of return times), and due to the influence of the saddle equilibrium point at the origin, it is not possible to find conditions in which all the UPOs become simultaneously entrained with the forcing (even for the natural frequency) at a fixed, established locking ratio (e.g., 1:1), but epochs of synchronized behavior (sometimes long) are interspersed with periods of time for which remains out of sync.

One of the findings of Refs. 12 and 13 is that the system actually exhibits synchronization at almost all time, but with time epochs characterized by different (alternating) locking ratios (that correspond to the number of turns of the chaotic oscillator with respect to the sinusoidal oscillator). Thus, phase slips due to imperfect phase synchronization exhibit distinctive features when compared to phase slips due to noise or eyelet intermittent behavior. This property of imperfect phase synchronization is very important when considering an experimental system (as is our case) subject to many sources of unavoidable experimental noise, like thermal noise, channel noise, etc. In this sense, we will show that the observed phase slips have a clear deterministic structure, corresponding to alternate locking ratios, quite different to the effect of external noise or proximity to the onset of the transition to phase synchronization.

Another point of interest in our study concerns the ability to model deterministic chaotic systems as it has been found that in some circumstances ${ }^{17,18}$ these systems may exhibit obstructions to deterministic modeling. Thus, in Ref. 18 the authors state that ... in laboratory experiments ( ..) it might only make sense to work directly with measured time series instead of a mathematical model when attempting to understand the long-term behavior of the system. These difficulties are a manifestation of nonhyperbolicity, and, from the reasoning above, they cannot be completely excluded in our system, namely when a system trajectory approaches the saddle equilibrium. In this sense, studying the phenomenon of imperfect phase synchronization in a real physical system is the only way of proving unambiguously its existence.

The goal of this paper is to present the first experimental study of imperfect phase synchronization for a circuit, that represents the Lorenz system subject to sinusoidal forcing. Section II discusses the Lorenz circuit and the experimental methodology. Section III discusses the main results of this work, and their comparison with the theoretical study. And, finally, Sec. IV contains the main conclusions of the present work.

\section{EXPERIMENTAL SYSTEM AND METHOD}

The analog circuit representing the Lorenz system ${ }^{11}$ is the one described in Refs. 19 and 20. Starting with the differential equations representing the Lorenz system plus a sinusoidal forcing term in the $\dot{z}$ term $^{12,13}$ (forcing is introduced in this term in order to preserve the symmetry of the equations),

$$
\begin{aligned}
& \dot{x}=\sigma(y-x) . \\
& \dot{y}=R x-y-x z, \\
& \dot{z}=x y-b z+E^{\prime} \sin \left(\Omega^{\prime} t\right) .
\end{aligned}
$$




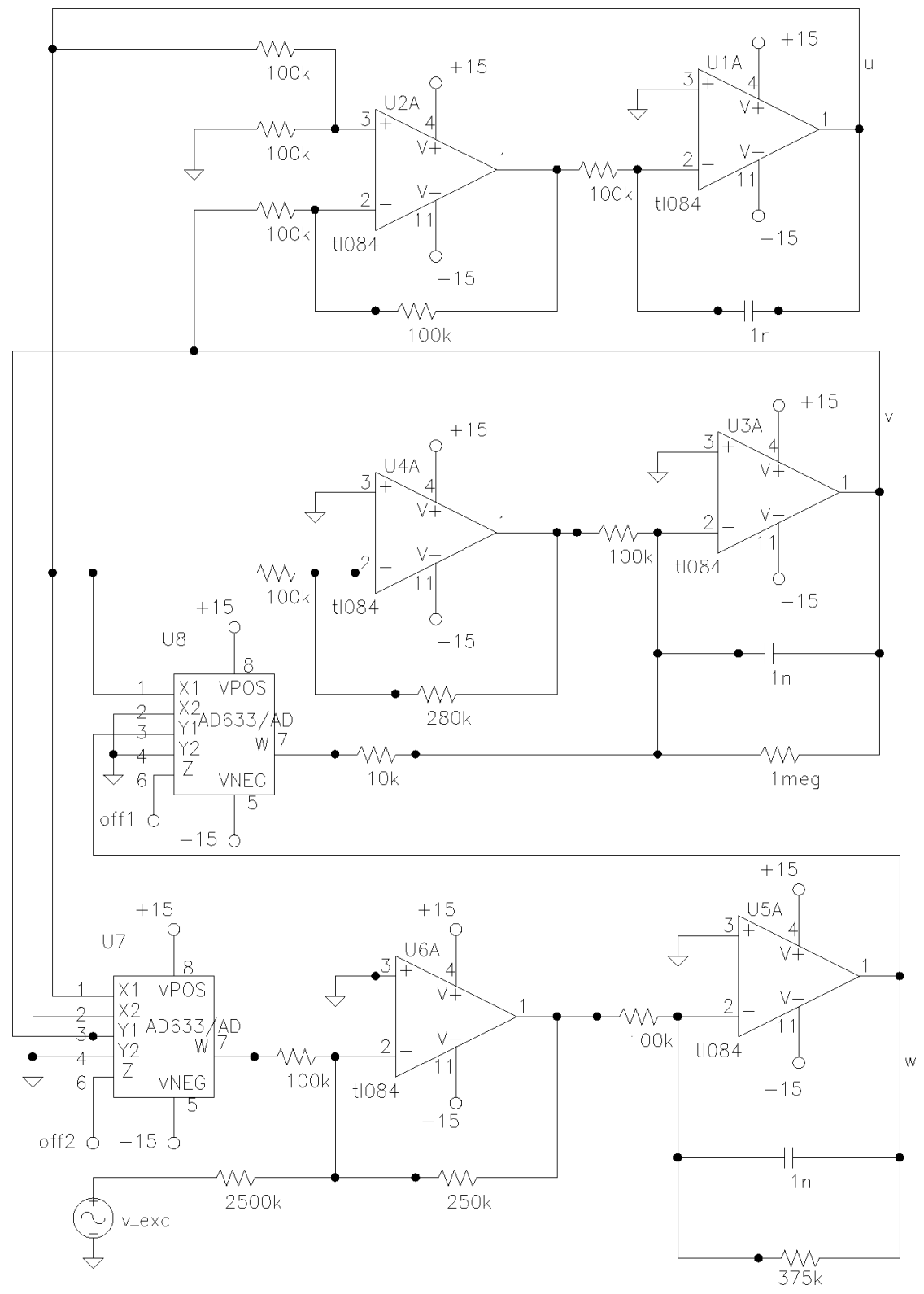

FIG. 1. Schematic representation of the circuit representing the Lorenz oscillator in rescaled variables, Eq. (3), including the sinusoidal forcing term.

The circuit consists of three integrators, one for each variable, and the nonlinear terms are represented using analog multipliers. The first step in designing the circuit is to rescale both the three state variables $x, y$, and $z$ in order to fit within the dynamical range of the source $[-15 \mathrm{~V}, 15 \mathrm{~V}]$, and such that the circuit operates in the frequency range of a few kilohertz. The transformation applied to the variables is the following:

$$
u=x / 5, \quad v=y / 5, \quad w=z / 10, \quad \tau=t / A, \quad A=10^{3} .
$$

This rescaling of variables leads to the following set of differential equations, in which the variables, $u, v, w$, are voltages across the three capacitors of the circuit, and in which the time is expressed in seconds,

$$
\begin{aligned}
& \dot{u}=A \sigma(v-u), \\
& \dot{v}=A(R u-v-10 u w), \\
& \dot{w}=A[(2.5 u v-b w)+E \sin (\Omega \tau)],
\end{aligned}
$$

where $E=E^{\prime} / 10$ and $\Omega=A \Omega^{\prime}=10^{3} \Omega^{\prime}$. In Eq. (3) the derivatives are with respect to $\tau$, while in Eq. (1) they are with respect to the original time $t$.

These equations have been implemented in an electronic circuit as shown in Fig. 1. The analog multipliers (AD633) have a noticeable offset at the output that may alter the dynamical behavior of the system, and this has been compensated using a compensation array. The tolerances of the resistors and capacitors are of $1 \%$ or less. In particular, the parameters for the Lorenz oscillator (3) recalculated from the actual values of the electronic components are as follows: $\sigma=10.19, b=2.664$, and $R=28.17$ (to be compared with the intended values: $\sigma=10, b=8 / 3$, and $R=28$ ). All the experimental results have been measured with a sampling rate of $80 \mathrm{kHz}$ using a data acquisition card with 12 bits of resolution, sufficient for the dynamic range of the Lorenz circuit. In all the studies presented here the amplitude of the forcing (3) has been fixed (through a resistance) to be $E=1 \mathrm{~V}$ [corre- 


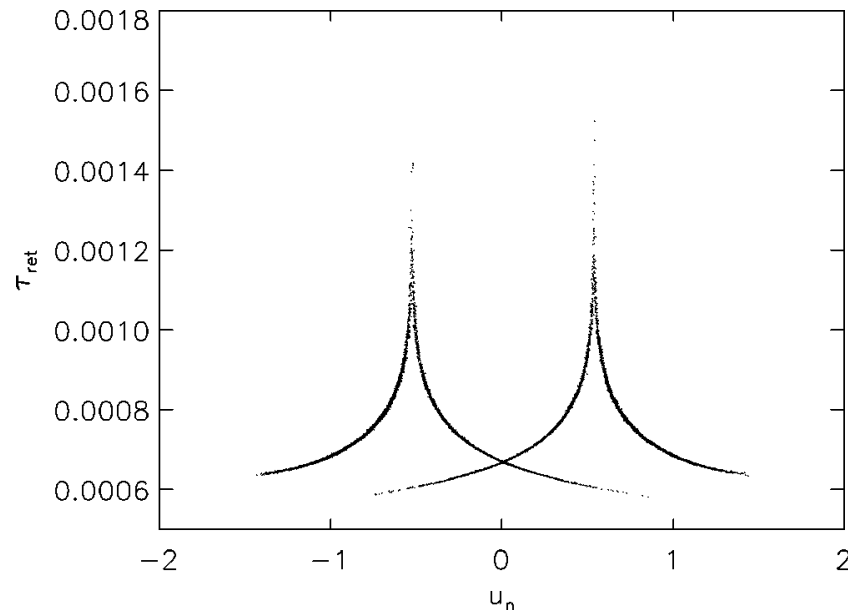

FIG. 2. Return time of the free running Lorenz oscillator at the Poincare surface $w=(R-1) / 10$ versus variable $u$. The two branches correspond to the two lobes of the attractor.

sponding to $E^{\prime}=10$ for the Lorenz system (1) before the rescaling]. Another important information concerning the system is the natural frequency of the unforced Lorenz system, that has been found to be $\omega_{0}=1311 \mathrm{~Hz}=8241 \mathrm{rad} / \mathrm{s}$. It has been estimated by using Eq. (2) in Ref. 13. The above quoted values of $\sigma, b, R$, and $E$ have been kept fixed in all the results presented in this paper.

\section{RESULTS}

As already mentioned in the introduction, the key feature of the Lorenz system for the parameter values considered in the present work is that the saddle point at the origin, $u=v$ $=w=0$ is part of the attractor. This single point is determinant in the dynamics of the system due to the fact that the dynamics of the Lorenz system for the parameter values studied in the present work consists basically in spiraling around one lobe followed by jumping to the other lobe, where the system exhibits the same spiraling dynamics, and jumping again. While the system is rotating in a given lobe these rotations are quite regular (and fast). Instead, jumping to the other (symmetric) lobe implies that the system becomes under the influence of the stable manifold of the saddle point at the origin, what leads to a slow down in the dynamics.

This behavior can be adequately characterized by taking a suitable Poincaré plane $10 w=z=R-1$, or $w=(R$ $-1) / 10$. The (high) rate of contraction along the transverse direction will lead to an approximately one-dimensional dynamics in this Poincare section. An interesting characterization of this behavior can be obtained by representing the return times at the Poincare cross section, i.e., the times that a trajectory spends between crosses with the Poincare cross section. As explained, these times are not bounded from above, and this can be also seen from Fig. 2, in which the time necessary to arrive to the Poincare cross section is represented versus the value of variable (voltage) $u$ at the crossing. From this representation it can be clearly seen that the return times diverge logarithmically when approaching the singularity.

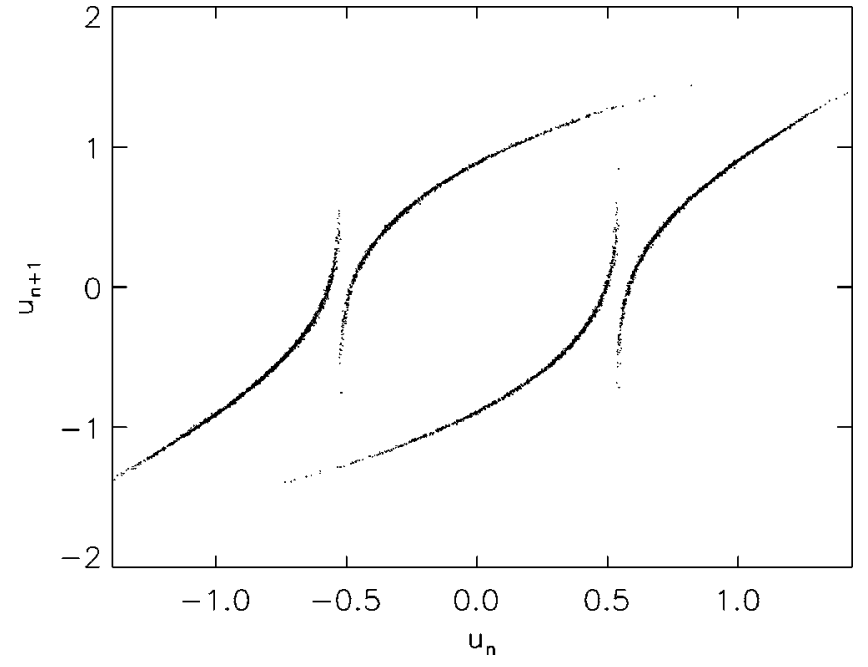

FIG. 3. Return map for the free running Lorenz oscillator at the Poincare surface $w=(R-1) / 10$. Variable $u$ at a given intersection with the Poincare surface, $u_{n+1}$, is plotted versus the same variable at the previous intersection, $u_{n}$. The two parts of the figure correspond to the two lobes of the attractor.

As the dynamics at the Poincare cross-section is approximately one dimensional, one could consider also a description based on iterated maps, namely by plotting variable $u$ at a crossing with the Poincare section versus $u$ at the preceding cross section (see Fig. 3). This representation will also exhibit a singularity, namely at the intersection of the Poincare cross section with the stable manifold of the saddle equilibrium.

As explained above the system studied in this work consists of an oscillator, that due to its chaotic dynamics exhibits a strong variation in the rotation period, forced by an oscillator rotating at a fixed pace. The most interesting dynamics of this system corresponds to those parameter values for which the system exhibits some kind of synchronization between these two different behaviors. The type of synchronization found can never be complete (due to the dissimilar nature of the systems involved), and it is rather phase synchronization. Thus, both types of oscillations (chaotic and regular) are different in detail, but beat at the same pace, what implies that they exhibit approximately the same frequency (this frequency is the average frequency in the case of the chaotic oscillator). This can be seen from Fig. 4, where the difference between the mean frequency of the Lorenz oscillator and the driving frequency is represented. For a fixed value of the forcing amplitude, $E=1 \mathrm{~V}$, and by varying the forcing frequency $\Omega$, a region in which the difference of frequencies is quite small (close to zero) can be found (cf. Fig. 4). A closer inspection (see the inset of Fig. 4) shows that the plateau is not exactly zero. The oscillations in the inset (compared to Fig. 11 in Ref. 13) should be ascribed to the larger number of turns used in the latter study, and also to experimental uncertainties. Anyhow, the frequency difference tends to be positive in all the synchronization range, as it should (cf. with Fig. 11 in Ref. 13).

Another quite interesting way of characterizing the imperfect phase synchronization behavior exhibited by our electronic sinusoidally excited Lorenz oscillator is by look- 


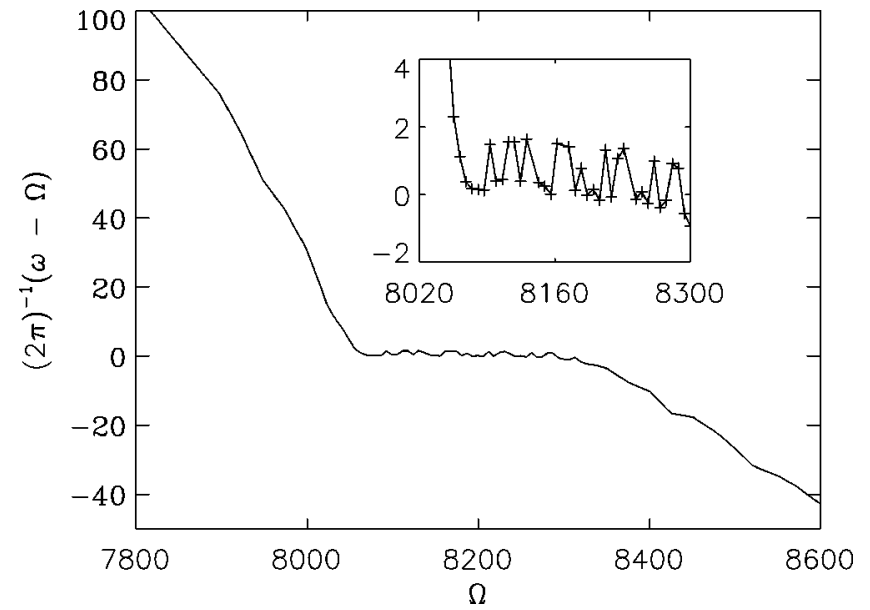

FIG. 4. Difference between mean frequency $\omega$ and driving frequency $\Omega$, in rad/s, estimated from a time series of $200 \mathrm{~s}$ for each value of the frequency, corresponding, approximately, to $2.6 \times 10^{5}$ turns of the chaotic oscillator.

ing at the attractor stroboscopically sampled at a suitable chosen Poincaré section (the result will be a snapshot attractor). In our case we consider the usual Poincaré section $z$ $=R-1$, that in rescaled units becomes $w=(R-1) / 10$, as explained above. The evolution of this snapshot attractor as the forcing frequency is varied can be seen in Fig. 5. The snapshot attractor exhibits a transformation from a diffuse cloud for frequencies of the sinusoidal oscillator outside the synchronization plateau of Fig. 4 to a well defined pattern inside this synchronization plateau, and, again, a diffuse cloud when increasing the forcing frequency outside the pla- teau [see Figs. 5(a)-5(f)]. However, (cf. also Ref. 13) even inside the well synchronized region the snapshot attractor never resembles a (more or less narrow) stripe as expected for the case of perfect phase synchronization (e.g., for the case of a phase coherent oscillator). As explained in Ref. 13 the well defined pattern obtained inside the synchronization plateau can be explained noticing that the system appears to spend most of the time in the central region (the figure is symmetric through the change $x \rightarrow-x$ due to the two lobes exhibited by the attractor), with occasional excursions that form the whiskers of the pattern.

Another demonstration of imperfect phase synchronization can be obtained by plotting the temporal development between the phases of the driven Lorenz system and the sinusoidal driving force (see Figs. 6 and 7). As explained above, imperfect phase synchronization is characterized by the unbounded character of return times, that leads to the driven system losing the pace of the sinusoidal generator. Thus, at first sight it can be surprising (e.g., from Fig. 7) that the phase slips (i.e., errors of synchronization) are quite often positive, as with the definition used this implies that the driven system actually performs more rotations than the sinusoidal driving (although in Fig. 6 one can find examples of both positive and negative jumps). The existence of these jumps (far from the transition to nonsynchronization) is one of the well known signatures of imperfect phase synchronization. ${ }^{12,13}$ It is also interesting to mention that although jumps by one turn, $2 \pi$ jumps in terms of phase, are the most common, $4 \pi$ can also be found (as in Fig. 7 for $t$ $\in[8,9])$. (a)

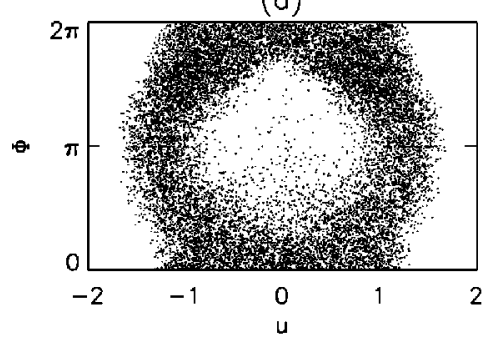

(c)

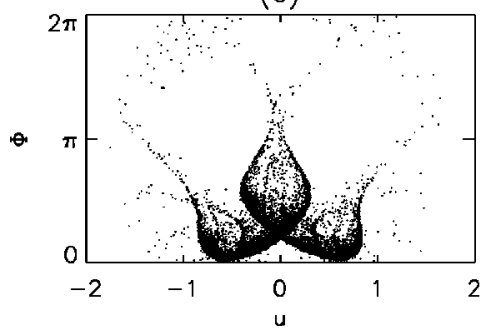

(e)

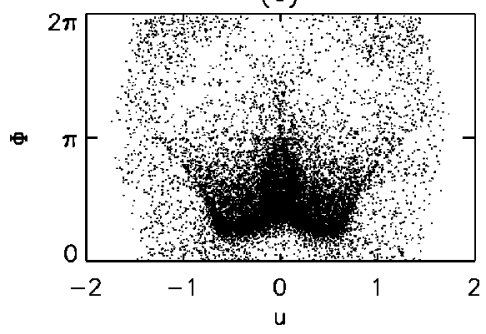

(b)

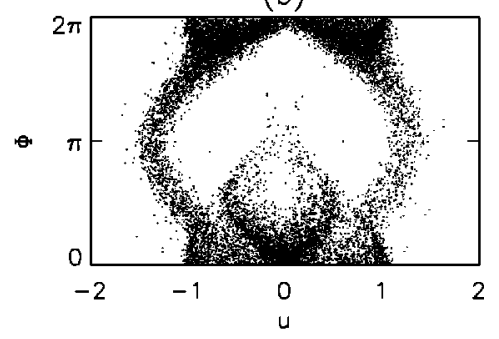

(d)

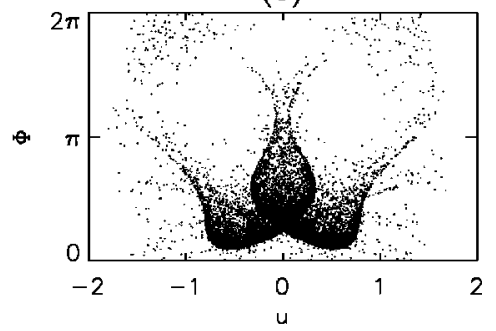

(f)

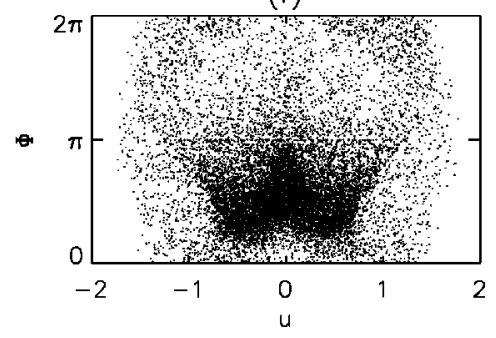

FIG. 5. Snapshot attractors of the Poincare mapping for different values of $\Omega$ : (a) $7350 \mathrm{rad} / \mathrm{s}$; (b) $7900 \mathrm{rad} / \mathrm{s}$; (c) $8150 \mathrm{rad} / \mathrm{s}$; (d) $8250 \mathrm{rad} / \mathrm{s}$; (e) $8350 \mathrm{rad} / \mathrm{s}$; (f) 8400 $\mathrm{rad} / \mathrm{s}$. The phase $\Phi$ of the sinusoidal oscillator at the Poincaré cross section $w=(R-1) / 10$ is represented versus variable $u$. 


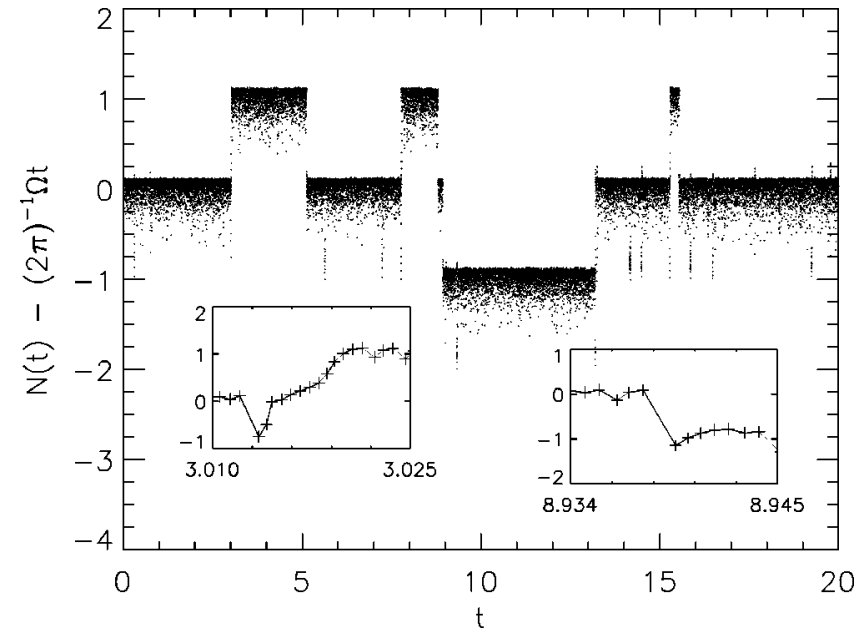

FIG. 6. Temporal development of the difference between the number of turns (rotations) of the Lorenz and sinusoidal oscillator, respectively, in the state of imperfect phase synchronization. $\Omega=8100 \mathrm{rad} / \mathrm{s}$, and crosses denote intersections with the Poincare cross section $w=(R-1) / 10$.

The above mentioned paradoxical fact that typically the driven Lorenz system performs more turns than the sinusoidal generator can be understood better by looking at some time traces of one of the three state space variables, e.g., $w$, and also at some state space projections (this is shown in Fig. 8). Considering variable $w$ has the advantage that it can be compared more cleanly with the sinusoidal pacemaker that is below in all the time traces (as $w$ remains always positive). Anyhow, one has to keep in mind that the oscillations with a large period are associated with changing lobe (moment where the dynamics is more influenced by the saddle equilibrium). The results in Fig. 8 correspond to three different phase jump events, that are the same presented in the three insets of Figs. 6 and 7: a positive and a negative, respectively, phase jump by $2 \pi$ in Fig. 6 and a positive $4 \pi$ jump in Fig. 7. As explained above, the three phase jumps have in common that they are preceded by a change of lobe in the

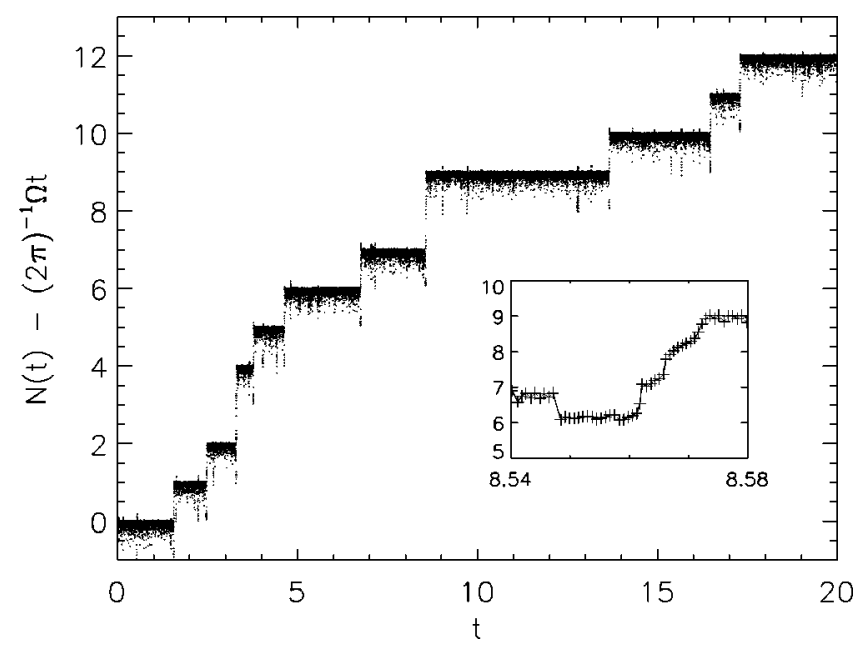

FIG. 7. Temporal development of the difference between the number of turns (rotations) of the Lorenz and sinusoidal oscillator, respectively, in the state of imperfect phase synchronization. $\Omega=8250 \mathrm{rad} / \mathrm{s}$, and crosses denote intersections with the Poincaré cross section $w=(R-1) / 10$.
Lorenz system (the slow turn in the left panels of Fig. 8), and so the driven Lorenz oscillator loses almost one turn when compared with the sinusoidal generator (this can also be seen clearly in the three insets in Figs. 6 and 7). Although the time from peak to peak (or, in other words, between two crossings through the Poincaré plane) is not the same (it varies chaotically), the variation happens in a relatively narrow range outside of these changes of lobe, and the Lorenz system is able to keep the pace with the drive. However, when one of these changes of lobe (and, thus, slow turns) occurs, the Lorenz system almost performs one turn less than the sinusoidal generator. These events are relatively common as can be seen from the cloud of points going down below the plateaus in Figs. 6 and 7, and that almost go down to the level of one turn less (with respect to the level at the plateau).

However, the absence of phase slips for many changes of lobe is due to the fact that the Lorenz system is able to perform an extra rotation, with respect to the sinusoidal generator. When this does not happen, a negative phase jump occurs, while sometimes the Lorenz system is, quite surprisingly, capable of performing two (or even three) extra turns. These fast rotations [some of them could even be called pseudo-rotations, as they are characterized by a very small rotation radius, see panels $(\mathrm{a}, 2)$ and $(\mathrm{c}, 2)$ in Fig. 8] may happen immediately after the change of lobe [panel $(\mathrm{a}, 2)]$, or slightly after [panel $(\mathrm{c}, 2)]$. Quite curiously, during these fast rotations the variable $w$ exhibits an interesting modulational, or beating, transient periodic behavior (resembling amplitude modulation). This behavior is probably associated to the interference between the anomalous fast rotations and the frequency of the sinusoidal generator (of course, as negative phase jumps do not have associated fast rotations, the system does not exhibit modulational behavior in this instance).

On the other hand, if one looks carefully at the behavior of the system in the intervals of time in which the system goes from an almost negative phase jump to a positive one (left inset of Fig. 6 and inset of Fig. 7) one can see that the driven Lorenz system performs more turns than the sinusoidal drive. Following Refs. 12 and 13 one can interpret this behavior by a change in the locking ratio between the drive and the Lorenz systems, that is no longer 1:1, but rather $n: n+2$ in a $2 \pi$ phase jump, or $n: n+3$ in a $4 \pi$ phase jump (the locking ratio will be $n: n+1$ in the frequent events in which the system does not exhibit a phase jump, although there is an almost negative phase jump, that occurs at almost all changes of lobe). In this sense, synchronization is not lost, but the system exhibits an alternation between different locking rates, in the periods of type in which the dynamics is more strongly nonhyperbolic (those in which the Lorenz system is under the effect of the saddle equilibrium point at the origin).

\section{DISCUSSION}

In the present study we have been able to characterize unambiguously imperfect phase synchronization in a sinusoidally forced representation of the Lorenz oscillator as an electronic (analog) circuit. The results presented in this con- 

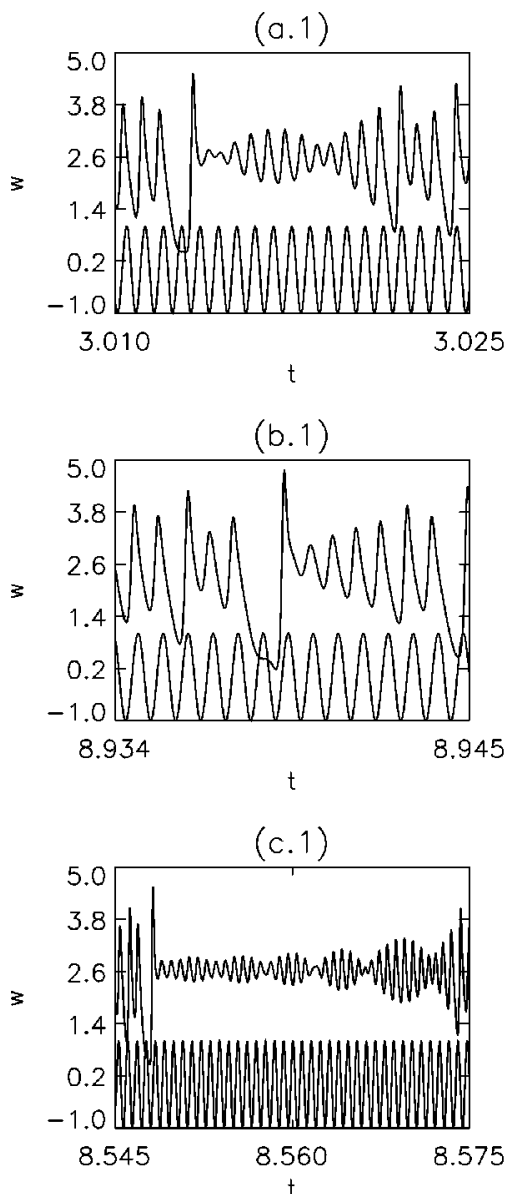
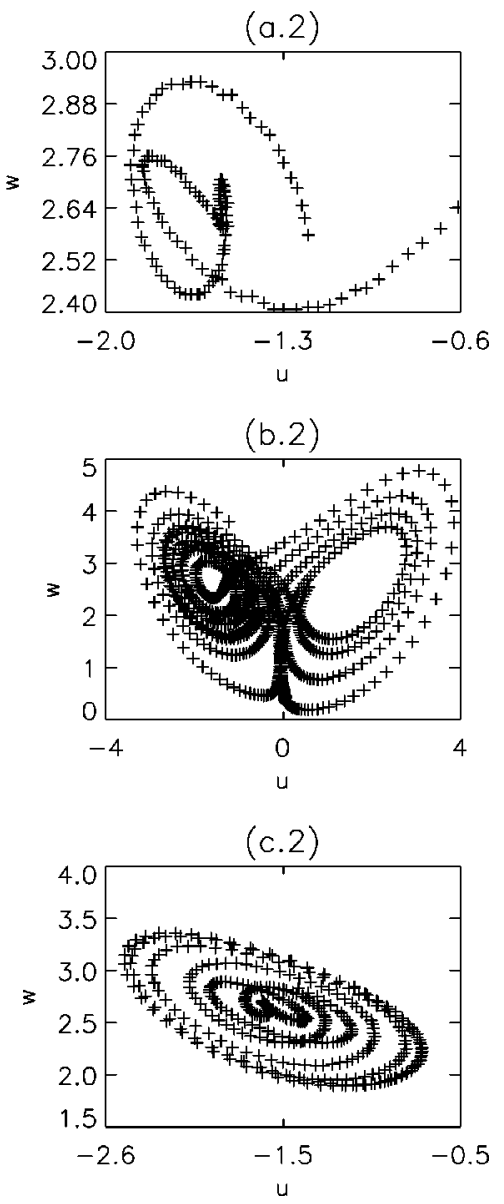

FIG. 8. Study of the behavior of the Lorenz system at three different phase jumps, namely those represented in three insets of Figs. 6 and 7. The three left panels contain the evolution of variable $w$ for the three phase jumps, respectively, while in the three right panels the phase portrait $w$ vs $u$ is represented for a subset of the time interval. Panel $(b, 2)$ represents the whole time snapshot in panel $(b, 1)$, i.e., $[8.934,8.945]$ while panels $(\mathrm{a}, 2)$ and $(\mathrm{c}, 2)$ detail the fast turns happening in the time intervals $[3.0135,3.01525]$ for $(\mathrm{a}, 1)$ and $[8.565,8.570]$ for $(\mathrm{c}, 1)$, respectively. tribution are so clear and clean that sometimes are almost identical to the equivalent results obtained from the direct numerical simulation of the dynamical system (cf. Refs. 12 and 13), even though in our case the system is subject to sources of noise (thermal, channel, tolerances in the components, etc.). This precise correspondence between experiment and numerical simulation makes us firmly believe that the imperfections observed in the phase synchronized state are not due to the presence of noise, proximity to the onset of phase synchronization or the like. In addition, the phase jumps have a clearly defined deterministic structure: during a transient period of time the system appears to be described by a different locking ratio (one would not expect this behavior in systems subject to noise or exhibiting intermittent bursts). The close correspondence between theory and experiment clearly confirms the reality of the phenomenon, and the possibility of modeling it theoretically.

Chaotic (perfect) phase synchronization was first demonstrated from the analysis of theoretical models, ${ }^{6}$ and later has been demonstrated through analog simulation of two coupled Rössler oscillators, ${ }^{21}$ and in some experimental physical systems: a plasma system ${ }^{22}$ and a chaotic laser array. ${ }^{23}$ Imperfect phase synchronization may be relatively common in dynamical systems with more degrees of freedom, and, in fact, in Ref. 13 it was argued that it could be the mechanism behind observations in some experimental data describing human cardiorespiratory activity. ${ }^{24,25}$
The outlook of the present experimental demonstration is that imperfect phase synchronization should be relatively common in a number of fields. The reason for this is that unstable fixed points being part of the closure of a chaotic attractor are relatively common in a number of fields, like fluid mechanics (e.g., in the transition to turbulence), nonlinear optics (e.g., semiconductor lasers), etc. However, the behavior of these systems can be more complex than the one presented here, as the unstable fixed point at the origin of the Lorenz system is a saddle, while higher-dimensional systems will typically have saddle-focus unstable fixed points.

\section{ACKNOWLEDGMENTS}

We acknowledge financial support from MCyT (Spain) and FEDER through Grants No. BFM2000-1108 and No. BFM2001-0341, and the European Commission through Projects No. OCCULT, No. IST-2000-29683 and No. HPRNCT-2000-00158. J.K. wishes to acknowledge the support of the Programa de Profesores Visitantes of Iberdrola.

\footnotetext{
${ }^{1}$ A. Pikovsky, M. Rosenblum, and J. Kurths, Synchronization: A Universal Concept in Nonlinear Science (Cambridge University Press, Cambridge, 2001).

${ }^{2}$ E. Ott, Chaos in Dynamical Systems (Cambridge University Press, Cambridge, 1993).

${ }^{3}$ H. Fujisaka and T. Yamada, Prog. Theor. Phys. 69, 32 (1983).

${ }^{4}$ L. M. Pecora and T. L. Carroll, Phys. Rev. Lett. 64, 821 (1990).
} 
${ }^{5}$ N. F. Rulkov, M. M. Sushchik, L. S. Tsimring, and H. D. I. Abarbanel, Phys. Rev. E 51, 980 (1995).

${ }^{6}$ M. G. Rosenblum, A. S. Pikovsky, and J. Kurths, Phys. Rev. Lett. 76, 1804 (1996).

${ }^{7}$ O. Rössler, Phys. Lett. A 57, 397 (1976).

${ }^{8}$ T. Yalcinkaya and Y.-C. Lai, Phys. Rev. Lett. 79, 3885 (1997).

${ }^{9}$ A. S. Pikovsky, M. G. Rosenblum, G. V. Osipov, and J. Kurths, Physica D 105, 219 (1997).

${ }^{10}$ K. Josic and D. J. Mar, Phys. Rev. E 64, 056234 (2001).

${ }^{11}$ E. N. Lorenz, J. Atmos. Sci. 20, 130 (1963).

${ }^{12}$ M. A. Zaks, E. H. Park, M. G. Rosenblum, and J. Kurths, Phys. Rev. Lett. 82, 4228 (1999).

${ }^{13}$ E. H. Park, M. A. Zaks, and J. Kurths, Phys. Rev. E 60, 6627 (1999).

${ }^{14}$ M. A. Zaks, E. H. Park, and J. Kurths, Int. J. Bifurcation Chaos Appl. Sci. Eng. 10, 2649 (2000).

${ }^{15}$ A. S. Pikovsky et al., Phys. Rev. Lett. 79, 47 (1997).
${ }^{16}$ A. Pikovsky et al., Chaos 7, 680 (1997).

${ }^{17}$ Y. C. Lai, C. Grebogi, and J. Kurths, Phys. Rev. E 59, 2907 (1999).

${ }^{18}$ Y. C. Lai and C. Grebogi, Phys. Rev. Lett. 82, 4803 (1999), and references therein.

${ }^{19}$ E. Sánchez, Ph.D. thesis, Universidad de Salamanca, Spain, 1999.

${ }^{20}$ E. Sánchez and M. A. Matías, Phys. Rev. E 57, 6184 (1998).

${ }^{21}$ U. Parlitz, L. Junge, W. Lauterborn, and L. Kocarev, Phys. Rev. Lett. 54, 2115 (1996)

${ }^{22}$ C. M. Ticos et al., Phys. Rev. Lett. 85, 2929 (2000).

${ }^{23}$ D. J. DeShazer, R. Breban, E. Ott, and R. Roy, Phys. Rev. Lett. 87, 044101 (2001).

${ }^{24}$ C. Schäfer, M. G. Rosenblum, H.-H. Abel, and J. Kurths, Nature (London) 392, 239 (1998)

${ }^{25}$ C. Schäfer, M. G. Rosenblum, H.-H. Abel, and J. Kurths, Phys. Rev. E 60, 857 (1999). 